\title{
Extreme-Scale $A b$ initio Quantum Raman Spectra Simulations on the Leadership HPC System in China
}

\author{
Honghui Shang* \\ SKL of Computer Architecture, \\ Institute of Computing Technology, \\ Chinese Academy of Sciences, \\ Beijing, China \\ shanghonghui@ict.ac.cn \\ Libo Zhang \\ National Supercomputing Center in \\ Wuxi, \\ China \\ zlb03@hotmail.com \\ Yangjun $\mathrm{Wu}$ \\ Institute of Computing Technology, \\ Chinese Academy of Sciences, \\ Beijing, China \\ wuyangjun21@163.com \\ Xin Liu \\ National Supercomputing Center in \\ Wuxi, \\ China \\ yyylx@263.net
}

\author{
Fang $\mathrm{Li}^{*}$ \\ National Supercomputing Center in \\ Wuxi, \\ China \\ 38349735@qq.com
}

You Fu

Shangdong University of Science and

Technology,

Qingdao, China

fuyou@sdust.edu.cn

Xiaohui Duan

Tsinghua University,

Beijing, China

sunrise_duan@126.com

Ying Liu

SKL of Computer Architecture, Institute of Computing Technology,

Chinese Academy of Sciences,

Beijing, China

liuying2007@ict.ac.cn

\author{
Yunquan Zhang* \\ SKL of Computer Architecture, \\ Institute of Computing Technology, \\ Chinese Academy of Sciences, \\ Beijing, China \\ zyq@ict.ac.cn \\ Yingxiang Gao \\ Institute of Computing Technology, \\ Chinese Academy of Sciences, \\ Beijing, China \\ 18810616698@163.com \\ Rongfen Lin \\ Tsinghua University, \\ Beijing, China \\ lrf21@mails.tsinghua.edu.cn
}

Dexun Chen

Tsinghua University, Beijing, China adch@263.net

\begin{abstract}
Raman spectroscopy provides chemical and compositional information that can serve as a structural fingerprint for various materials. Therefore, simulations of Raman spectra, including both quantum perturbation analyses and ground-state calculations, are of significant interest. However, highly accurate full quantum mechanical (QM) simulations of Raman spectra have previously been confined to small systems. For large systems such as biological materials, full QM simulations have an extremely high computational cost and remain challenging. In this work, robust new algorithms and advanced implementations on many-core architectures are employed to enable fast, accurate, and massively parallel full $a b$ initio simulations of the Raman spectra of realistic biological systems containing up to 3006 atoms, with excellent strong and

\footnotetext{
${ }^{*}$ Corresponding authors

Permission to make digital or hard copies of all or part of this work for personal or classroom use is granted without fee provided that copies are not made or distributed for profit or commercial advantage and that copies bear this notice and the full citation on the first page. Copyrights for components of this work owned by others than ACM must be honored. Abstracting with credit is permitted. To copy otherwise, or republish, to post on servers or to redistribute to lists, requires prior specific permission and/or a fee. Request permissions from permissions@acm.org.

SC '21, November 14-19, 2021, St. Louis, MO, USA

(C) 2021 Association for Computing Machinery.

ACM ISBN 978-1-4503-8442-1/21/11 . \$ \$15.00

https://doi.org/10.1145/3458817.3487402
}

weak scaling. Up to a performance of $468.5 \mathrm{PFLOP} / \mathrm{s}$ in doubleprecision and $813.7 \mathrm{PLOPS} / \mathrm{s}$ in mixed-half precision is achieved on the new-generation Sunway high-performance computing system, suggesting the potential for new applications of the QM approach to biological systems.

\section{KEYWORDS}

Quantum mechanics, Raman spectra, Massively parallel and highperformance simulations, All-electron, Many-core processor, Biological systems

\section{ACM Reference Format:}

Honghui Shang, Fang Li, Yunquan Zhang, Libo Zhang, You Fu, Yingxiang Gao, Yangjun Wu, Xiaohui Duan, Rongfen Lin, Xin Liu, Ying Liu, and Dexun Chen. 2021. Extreme-Scale $A b$ initio Quantum Raman Spectra Simulations on the Leadership HPC System in China . In The International Conference for High Performance Computing, Networking, Storage and Analysis (SC '21), November 14-19, 2021, St. Louis, MO, USA. , 13 pages. https://doi.org/10.1145/ 3458817.3487402

\section{JUSTIFICATION FOR ACM GORDON BELL PRIZE}

This study has achieved unprecedented full $a b$ initio Raman spectra simulations of real biological proteins with up to 3006 atoms. Using the new-generation Sunway high-performance computing system, 
up to 468.5 PFLOP/s double precision and $813.7 \mathrm{PFLOP} / \mathrm{s}$ mixed-half precision performance can be achieved, with near-linear strong and weak scaling.

\section{PERFORMANCE ATTRIBUTES}

\begin{tabular}{c|c}
\hline \hline $\begin{array}{c}\text { Category } \\
\text { of achievement }\end{array}$ & $\begin{array}{c}\text { Scalability } \\
\text { Peak Performance }\end{array}$ \\
\hline Type of method used & Non-linear equations, Explicit \\
\hline $\begin{array}{c}\text { Results reported on } \\
\text { the basis of }\end{array}$ & Whole application including I/O \\
\hline Precision reported & Double precision, Mixed precision \\
\hline System scale & Measured on Full System \\
\hline Measurement mechanism & Timers, FLOP count \\
\hline \hline
\end{tabular}

\section{OVERVIEW OF THE PROBLEM}

Raman spectroscopy is a very powerful analytical tool for materials science, allowing the effective extraction of chemical and structural information. The principle behind Raman spectroscopy is the Raman effect. This was discovered in 1928 by Chandrasekhara Venkata Raman, who performed experiments in which green light was shone into a chloroform solution and a weak yellow light was emitted as a result. This clearly indicated that some of the green light was being scattered inelastically by the molecules in the solution. This inelastic scattering is known as the Raman effect, and its discoverer was rewarded with the Nobel Prize for Physics. In fact, this inelastic scattering that appears in a unique Raman spectrum is caused by the interactions between the light and the vibrations of the chemical bonds. As a result, the chemical composition and structure of materials can be identified from the spectra. There are many advantages to Raman spectroscopy: there is no interference from water molecules, the samples are not damaged, the target molecules can be identified with a short detection time and there is good sensitivity to small changes in material structure. Hence, there have been many applications of Raman spectroscopy in physics, chemistry, materials science, and biomedical research. As shown in Fig. 1, when using Raman spectroscopy to characterize a biological molecule, the biochemical and structural information can usually be obtained without labeling, and whole biochemical processes, such as metabolic pathways or dynamics, can be identified. As Sunzi once said, "Know thy self, know thy enemy. A thousand battles, a thousand victories." The valuable structural information extracted from Raman spectra gives us the opportunity to know our enemy (e.g., SARS-CoV-2), enabling the further development of therapeutic countermeasures.

In principle, Raman spectroscopy provides information about atomic vibrations. To fully understand the information provided by Raman spectroscopy, parameter-free $a b$ initio investigations are essential, because they provide a direct link between the atomic structure and the spectral features. This is an invaluable source of structural information, as well as the basis for improving the fundamental understanding of the physical mechanism between atoms.

However, the $a b$ initio simulation of Raman spectra requires the calculation of up to the third-order derivative of the total energy, in which the calculation of electric field and atomic displacement perturbation needs to be considered at the same time. The calculation of the analytical energy derivative is not trivial, and the

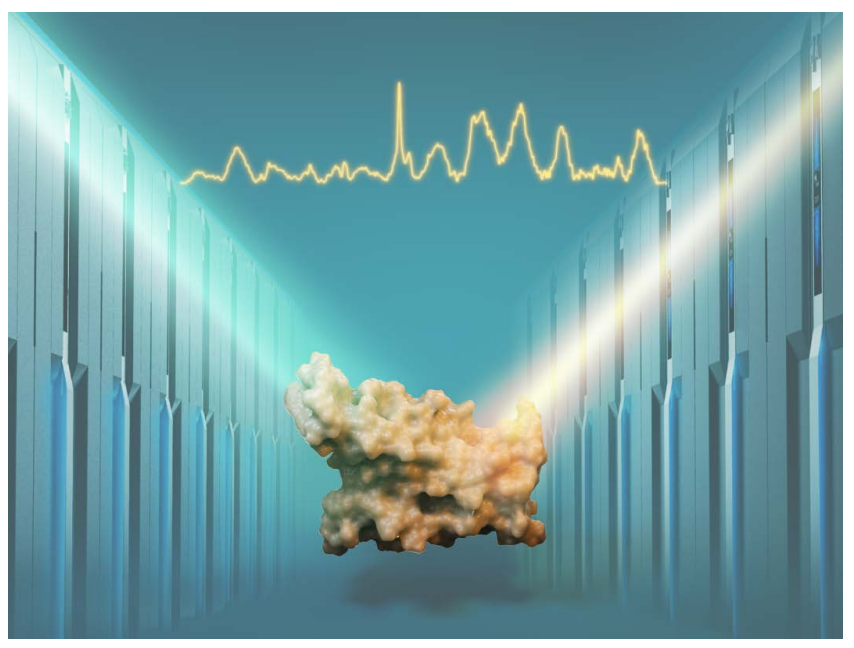

Figure 1: Principles of Raman spectra, reflecting the intrinsic biochemistry information of the protein.

density-functional perturbation theory (DFPT) beyond the standard density-functional theory (DFT) is required to obtain the high-precision results. The DFPT method can directly simulate the Raman spectroscopy, providing information about the atomic structure, vibrations, and the dynamical process. Since the perturbations in DFPT destroy the boundary conditions of periodic systems, and the atomic displacements cause a change of the entire basis set, the construction of the related matrix elements in DFPT is complex. Therefore, compared to DFT, the parallelization and corresponding optimizations in DFPT are much more complicated. As a result, the computation of Raman spectra has traditionally been limited to small systems, and remains challenging for large systems consisting of up to several thousand atoms. Therefore, a highly efficient acceleration of $a b$ initio simulation on modern heterogeneous many-core supercomputers is urgently needed. In this study, we optimize the FHI-aims [1] software on the new-generation Sunway heterogeneous many-core supercomputer to address this challenge.

FHI-aims [1] is a massively parallel package for computational molecular and materials science. It contains state-of-the-art DFPT computation modules $[17,18]$ that can directly link experimentally measured physical properties (e.g., phonon dispersions, polarizability, Raman spectra) with the quantum response or perturbation of the system. In this paper, we describe a robust new algorithm and advanced implementation of FHI-aims that enables the full quantum mechanical (QM) calculation of Raman spectra to be scaled up to an unprecedented level of 3006 atoms. The package is implemented on the new-generation Sunway heterogeneous many-core supercomputer without introducing any approximations to the QM calculations, beyond those inherent to DFT/DFPT. The optimization methods proposed in this work could be extended to other DFPT codes with the same computational characteristics. Hence, they will be broadly beneficial to the quantum chemistry, biological, and materials science communities.

Based on these developments, highly accurate all-electron $a b$ initio calculations could be used to understand the structural information of biological systems. This work provides a starting point 
Table 1: Summary of existing works on $a b$ initio simulations for different orders of total energy on supercomputers. Values taken from the corresponding papers. Unreported values are labeled as '-'. For numerical methods, PP refers to pseudopotential, AE (FP) refers to all-electron (full potential) method. For the basis functions, PW refers to plane wave, GTO refers to Gaussian-type orbital, PAO refers to pseudo-atomic orbital, blip refers to B-spline function, psinc refers to periodic sinc function, NGWF refers to non-orthogonal generalized Wannier function, SF refers to localized support functions, NAO refers to numerical atomic orbital. For the physical properties, $\mathrm{E}^{(0)}$ denotes the total energy of the ground state, $\mathrm{E}^{(1)}$ (energy first derivative) denotes force of individual atoms so that we can see the dynamic progress, $E^{(2)}$ (energy second derivative) and $E^{(3)}$ (energy third derivative) denote the response of the system with respect to the external perturbation (e.g., electric field), which need to be evaluated with density-functional perturbation theory (DFPT).

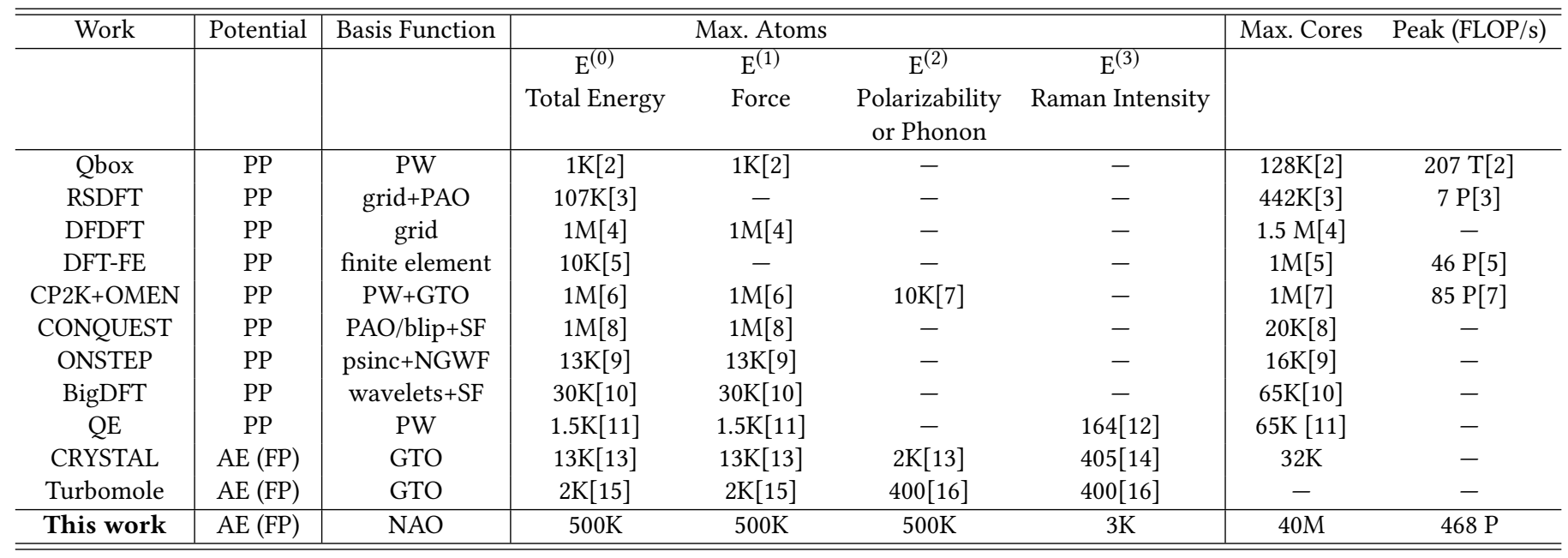

for the application of full QM approaches to structural studies of biological systems, which would provide a fundamental understanding of the interactions between the atoms within them.

\section{CURRENT STATE OF THE ART}

In order to calculate the above-mentioned physical properties theoretically, the quantum perturbation form of the Schrödinger equation needs to be solved numerically within the DFPT framework. In the DFPT approach, the many-body problem is simplified by a single-particle approximation; then, the single-particle wave functions are expressed as a linear combination of predefined basis functions. Thus, we can obtain a matrix equation to be solved numerically. The basis functions can be plane waves (PWs), as used in Quantum ESPRESSO (QE) [19], VASP [20], ABINIT [21] and Qbox [2] codes, the basis set can also be the uniform realspace grids as used in Octopus [22], or the periodic sinc functions as used in ONETEP [9], or the b-spline functions as used in CONQUEST [8], or the finite elements used in DFT-FE [5], or the wavelets used in BigDFT [10], or mixed Gaussian and PWs as used in CP2K [23]. Although the above basis sets can be converged systematically, the oscillatory behavior near the atomic nucleus cannot be accurately represented because of the excessive computational demands (e.g., $10^{5} \mathrm{PWs}$ are needed for one core orbital). As a result, when using these basis sets, pseudization methods [24] using pseudo-potentials or projector-augmented waves have been introduced, in which the core potential is replaced by a "fake" one. Although the pseudo-potentials have been carefully constructed to ensure that the valence part remains consistent with the all-electron method, core-shell information remains missing. To consider the core and valence states on an equal footing and achieve better precision compared with the pseudization method [24], all-electron approaches have been developed, e.g., the all-electron Gaussian atomic orbital method in Gaussian [25], CRYSTAL [13], and the all-electron numerical atomic orbitals method in DMol [26] and FHI-aims [1].

To date, most DFPT studies of physical properties (e.g., Raman spectra) have focused on small systems with only up to several hundred atoms because of the high computational demands involved. To the best of our knowledge, the largest full ab initio Raman spectra simulation included around 400 atoms $[14,16]$ using a Gaussian basis set. A much larger system is needed to access new physical phenomena. The fragment method [27, 28] was developed to deal with large systems (around 1000 atoms). However, the fragments need to be carefully chosen to reduce interpretation errors $[29,30]$ and the fragment needs to be extended to the whole system for the simulation of very delocalized modes in the supercell [28].

Overall, the current state-of-the-art DFPT methods for largescale systems are limited with respect to either accuracy or computational efficiency and scalability. Thus, DFPT calculations on large-scale systems are severely limited. This represents the key bottleneck in simulating the physical properties of real systems. The performance summary of exiting works is give in Table 1, where the maximum number of atoms that can be simulated for different physical quantities (different orders of total energy) is provided.

In this study, we use the DFPT modules implemented in the all-electron full-potential massively scalable FHI-aims package [1] 
to demonstrate that we can perform full QM calculations of the Raman spectra of real biological systems containing up to 3006 atoms. The calculations are implemented using massively parallel algorithms on the new-generation Sunway supercomputer without introducing any approximations to the QM calculations, beyond those inherent to DFT/DFPT.

In the following, the development of the DFPT method in FHIaims is described in detail. Before the DFPT calculation, the normal DFT calculation needs to be performed to solve the Kohn-Sham (KS) single-particle equations:

$$
\hat{h}_{\mathrm{KS}}\left|\psi_{i}\right\rangle=\left[\hat{t}_{s}+\hat{v}_{\text {ext }}(r)+\hat{v}_{H}+\hat{v}_{x c}\right]\left|\psi_{i}\right\rangle=\epsilon_{i}\left|\psi_{i}\right\rangle,
$$

where the first term on the left-hand side is the KS Hamiltonian $\left(\hat{h}_{K S}\right), \hat{t}_{s}$ represents the single-particle kinetic operator of the electron, $\hat{v}_{\text {ext }}$ is the (external) electron-nuclear potential, $\hat{v}_{H}$ is the Hartree potential, and $\hat{v}_{x c}$ represents the exchange-correlation potential. The KS single-particle states $\psi_{i}$ and their eigenenergies $\epsilon_{i}$ can be calculated by solving Eq. (1). If an external electric field $\mathbf{E}=\left(e_{x}, e_{y}, e_{z}\right)$ of strength $e_{\gamma}$ is applied to the system ( $\gamma$ refers to different cartesian axis), the KS Hamiltonian gains an additional term, $\hat{h}_{K S}^{(1)}=\hat{h}_{E}=-\mathbf{r} \cdot \mathbf{E}$. Thus, we obtain a perturbative version of the KS equation, which is called the Sternheimer equation:

$$
\left(\hat{h}_{K S}-\epsilon_{i}\right)\left|\psi_{i}^{(1)}\right\rangle=-\left(\hat{h}_{K S}^{(1)}-\epsilon_{i}^{(1)}\right)\left|\psi_{i}\right\rangle .
$$

This equation can be solved self-consistently to give the corresponding first-order density, where $f_{i}$ denotes the occupation number of eigenstate $\psi_{i}$ :

$$
\begin{aligned}
n(\mathbf{r})^{(1)} & =\frac{\partial n^{(0)}(\mathbf{r})}{\partial e_{\delta}} \\
& =\sum_{i} f_{i}\left[\psi_{i}^{*(0)}(\mathbf{r}) \psi_{i}^{(1)}(\mathbf{r})+\psi_{i}^{*(1)}(\mathbf{r}) \psi_{i}^{(0)}(\mathbf{r})\right]
\end{aligned}
$$

We then obtain the polarizability, which corresponds to the second-order derivative of the total energy with respect to the external electric field:

$$
\alpha_{\gamma \delta}=\int r_{\gamma} \cdot \frac{\partial n^{(0)}(\mathbf{r})}{\partial e_{\delta}} d \mathbf{r} .
$$

After the polarizability $(\alpha)$ has been determined using the analytical DFPT approach, the Raman intensity can be calculated. The Raman intensity is proportional to the square of the third-order derivative of the total energy with respect to both the external electric field and the atomic displacement:

$$
I_{\text {Raman }}\left(\omega_{p}\right) \propto\left(\alpha_{i j}^{\prime}\right)_{p}^{2}
$$

where $\left(\alpha_{i j}^{\prime}\right)_{p}=\left(\partial \alpha_{i j} / \partial Q_{p}\right)=\left(\sum_{I} e_{I, p} \partial \alpha_{i j} / \partial R_{I}\right)$ is the derivative of the $i j$-th component of the polarizability with respect to the displacement of normal phonon mode $e_{p}$. These derivatives are computed using finite differences; we evaluate the polarizability tensor using DFPT [18] at $6 \mathrm{~N}$ nuclear displacements in the unit cell around the equilibrium position ( $3 N$ forward and $3 N$ backward), where $N$ is the number of atoms per unit cell. The atomic derivatives are then multiplied by the phonon eigenvectors to give $\left(\alpha_{i j}^{\prime}\right)_{p}$ for the calculation of the Raman intensity.

A flowchart of the Raman spectra simulation with DFPT in FHIaims is shown in Fig. 2. After the ground state calculation with DFT has been completed, the DFPT cycle begins, using an initial guess for the response of the density matrix $P^{(1)}$, which then allows the response density $n^{(1)}(\mathbf{r})$ to be constructed. The associated response of the electrostatic potential $V_{e s, t o t}^{(1)}(\mathbf{r})$ is calculated by solving the Poisson equation in real space. The response Hamiltonian $H^{(1)}$ is calculated with the response density and potential. In turn, all these ingredients enable us to set up the Sternheimer equation to get the response density matrix $P^{(1)}$. We iteratively repeat the DFPT loop until self-consistency is reached, i.e., until the changes in $P^{(1)}$ become smaller than a user-defined threshold. Finally, the physical properties (e.g. polarizability, Raman spectra) are evaluated using the converged response density matrix.

\section{INNOVATIONS REALIZED}

\subsection{All-electron grids and the 3 level parallelization strategy}

FHI-aims uses all-electron all-potential numerical atomic basis functions to achieve high-precision results. In this scheme, the all-electron atomic orbitals are discretized using an atom-centered grid [31], as illustrated in Fig. 2 (c), in order to treat all-electron full-potential systems where the integrand is dominated by cusps at atomic nuclei. This atom-centered grid is first partitioned for each atom, and then the single-center (atom) grids are further separated into radial and angular parts. In this work, all the perturbation properties are calculated within such discretized three-dimensional physical grids, which are suitable for massively parallel implementations.

In order to partition non-even grid meshes in an efficient way, the grid-adapted cut-plane method is used to form batches [32], as indicated by the different color boxes in Fig. 2 (c). The procedure to obtain batches is as follows: first, the centers of mass for all points are computed; second, the direction of the cut-plane is determined by computing the normal of the plane; third, the position of the cut-plane is computed to divide all the points into two even-sized sets, and the full points are split into two subsets (batches) using the cut-plane. The above procedure is performed recursively until all the batches are the desired size (around 100 to 300 points per batch).

The load balancing for integration is achieved by eventually distributing the integration points over the MPI processes. The batches/grids are distributed according to the current summation of the points in each process; the new batch is always sent to the process with the minimal number of points. In this way, load balancing for grid integration is achieved. During an integration calculation, e.g., that of the response Hamiltonian matrix, each batch is calculated locally for one part of the matrix element, and finally the matrix elements from all processes are combined. There is another adaptive load balancing algorithm, in which, the running time and spatial location of each batch are collected in the first DFT/DFPT iteration, and the distribution of the batches over the process is calculated based on the location and running time. The distance between batches within one process is as small as possible, and the total running time for each batch is as close as possible. The batch distribution of the remaining iterations is basically the same as that of the first iteration, so it can reduce the overhead caused by the unbalanced distribution. This adaptive load balancing 

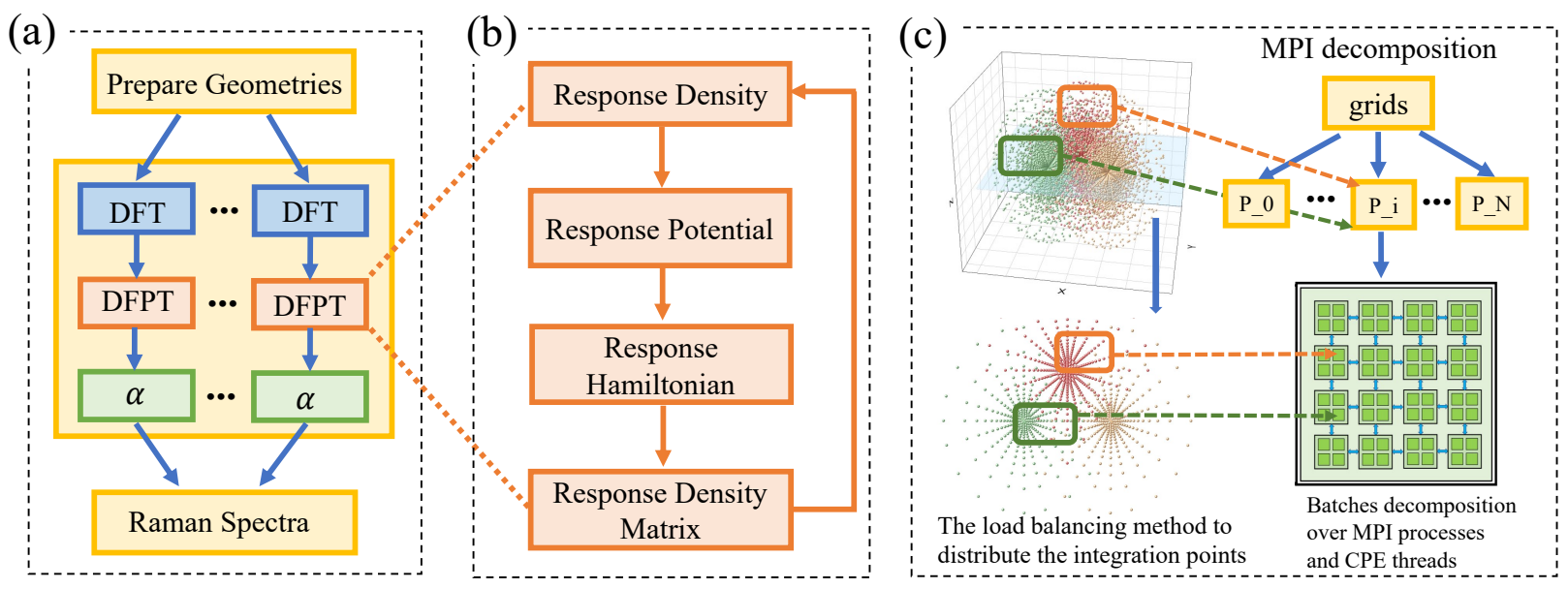

Figure 2: (a) Flowchart of the Raman spectra simulation with density-functional perturbation theory (DFPT). (b) The flowchart for DFPT with the all-electron approach. (c) The process-level and thread-level parallelization for the non-uniform grid integration, the grids in one batch are distributed into the threads to provide many-core acceleration. Three-dimensional illustration of the grid distribution of the $\mathrm{H}_{2} \mathrm{O}$ molecule is shown, the batches are indicated by different color boxes.

algorithm could reduce the number of splines calculation and the computational efficiency can be further improved.

Three levels of parallelization are used for Raman spectra calculations. As shown in Fig. 2 (a), the calculation of the polarizabilities can be performed in an embarrassingly parallel manner with different geometries, that is, the first level of parallelization. As no communication between the polarizability calculations is required, we split the whole CPU pool into different sub-groups and sub-communicators. Within each sub-group, the polarizability is calculated with the DFPT method. Within each DFPT calculation, another two levels of parallelization have been adopted for calculation of the numerical integration. The first level of parallelization for DFPT is performed over the batches, which are distributed across all MPI processes. This enables good parallel scalability using the adaptive batch distribution algorithm to achieve load balance. The second level of parallelization for DFPT is again performed over the batches within one process. Acceleration on threads can further improve the performance.

\subsection{Many-core Optimization Strategies}

We ported all the computation kernels into the new-generation Sunway processor. As the calculation of the response density and response potential are distributed over the grid points, there is no communication between the processes and threads during the computation. We perform DMA tiling to efficiently access the main memory; we also adopt double buffering to overlap the computations with memory accesses.

Loop tiling is an important transformation for exploiting the spatial and temporal locality of data accesses in loop nests. Previous work $[33,34]$ has demonstrated the use of the loop tiling model for such scratch pad memory (SPM)-based architectures. For example, we allocate static space on SPM and apply static loop tiling to keep the blocks of regularly access the arrays, e.g. value_1, as shown in Fig. 3 (a).
After determining the loop tiling strategy to efficiently utilize the on-chip SPM, we need to fetch data from the main memory and pass it to the SPM in an efficient manner. A computer processing element (CPE) has two execution pipelines, $P 0$ and $P 1$. $P 0$ supports scalar and vectorized computing operations of both floating-point and integer types, whereas $P 1$ supports scalar and vectorized data load/store, compare, and jump operations, and scalar integer operations. The two pipelines provide an opportunity for data access and computation operations to be overlapped [35]. For this purpose, we leverage double buffering [36] to overlap the data transfer and the on-chip computation. The double buffering process is shown in Fig. 3(d).

To further improve the computation efficiency of each kernel, we also exploit the vectorization (SIMD operations) to the functions. The new-generation Sunway CPU provides a word vector shuffling interface, which supports the full shuffling of 512-bit vector registers in CPE, as shown in Fig. 3(b). The parallel look-up table instruction is also offered, that the LDM can be divided into 16 slots, the size and width of each slot are $16 \mathrm{~KB}$ and $32 \mathrm{Bit}$. For a vector in register, the result of parallel table lookup consists of the elements in different slots in LDM, while the vector obtained by vector loading is often composed of elements in a continuous memory space, the vector loading and parallel look-up instruction are shown in Fig. 3(c). The MPE-CPE collaboration is another way to improve the computational efficiency, as shown in Fig. 3(e), while the MPE performs the calculation for "kernel 1", the CPE executes the code "kernal2", which realizes the mutual hiding of calculations in the MPE and CPE.

In the calculation of the response potential, we apply kernel fusion to reduce the overhead of multiple kernel launching, as shown in Fig. 3(f), after kernel fusion, the data can only be fetched from the main memory once. 
(a)
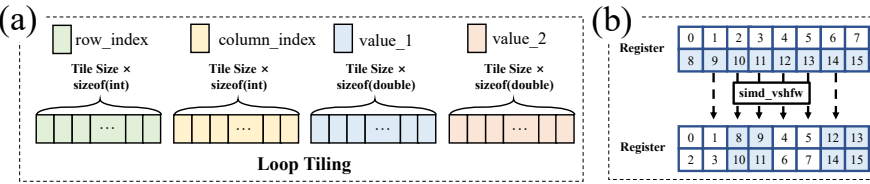

(c)

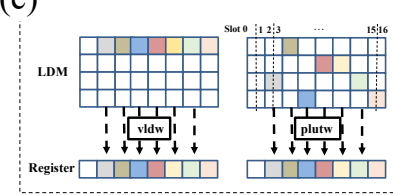

(d)

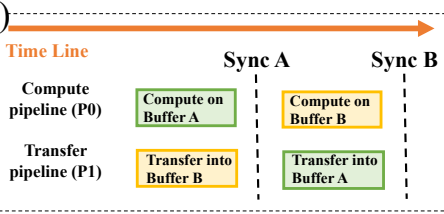

(e)

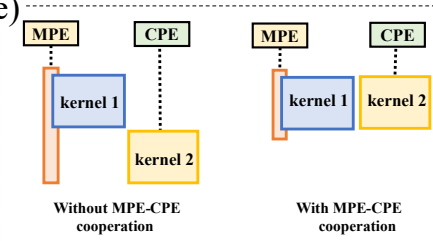

(f)

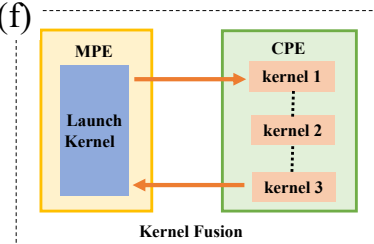

(g)

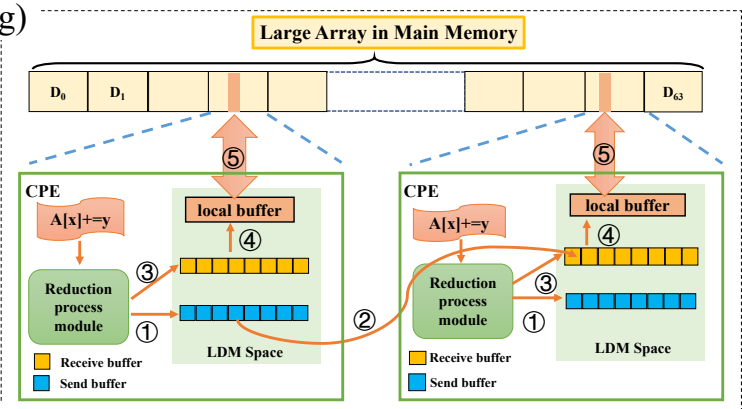

(h)

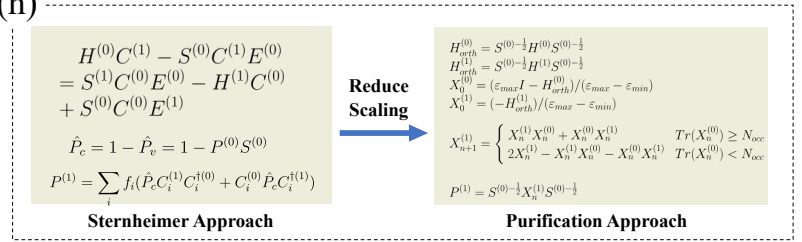

Figure 3: Illustration of the optimizations procedures. (a) DMA loop tiling; (b) Word vector shuffling instruction. (c) Vector loading and parallel lookup table instructions. (d) Double buffering. (e) MPE-CPE collaboration. (f) Kernel fusion. (g) Large array reduction algorithm. (h) Explicit purification algorithm for reducing the computational scaling.

In the calculation of the response Hamiltonian, there is an integration over the grid as follows:

$$
H_{\mu, v}^{(1)}=\int \chi_{\mu}(\mathbf{r}) \hat{h}_{K S} \chi_{v}(\mathbf{r}) d \mathbf{r} .
$$

After evaluating the integration over every $\mathrm{CPE}$, the Hamiltonian elements are summed up using a large array reduction, which can be written as follows:

$$
a r r_{i}=\operatorname{sum}\left\{v a l_{j} \mid i d x_{j}=i\right\}
$$

where arr is a large array, and val is irregularly distributed. In practical applications, codes like $\operatorname{arr}[i d x[j]]+=v a l[j]$ often occur; because the capacity of local data memory (LDM) is limited, the size of array arr exceeds the LDM capacity, and the traditional specification method is no longer applicable. However, if arr units in main memory are updated directly, write conflicts between CPEs will occur, and CPE locks need to be used to ensure that the write operation is correct. In this case, $64 \mathrm{CPEs}$ contend for the same lock and $a r r$ to access main memory directly, which can seriously affect the performance of the reduction operation. Therefore, we designed an efficient distributed reduction based on the RMA communication mechanism between CPEs. The schematic diagram of this method is shown in Fig. 3(g). The principle of this method is as follows: the large array arr that needs to be contracted is divided into 64 pieces of data, and $64 \mathrm{CPEs}$ are responsible for the contracted operation for each piece of data, respectively. Each CPE applies a temporary buffer buf on LDM to buffer the large data blocks for which it is responsible. Besides, each slave core creates 64 send buffer units (denoted $\mathrm{S} 0, \mathrm{~S} 1, \ldots, \mathrm{S} 63$ ) and 64 receive buffer units (denoted R0, R1,.., R63), which are used to send and receive the RMA messages of 64 slave cores corresponding to the reduction operation, respectively. The reduction operation is performed as follows,

- Step 1: According to the current $i d x$ value, calculate the location of the element to be updated in the CPE managed data interval (assuming the destination CPE ID is $n$ ), and then cache the reduction value and the reduction operator to the local sending cache of the corresponding CPE's buffer unit $S_{n}$.

- Step 2: Check whether the sending buffer unit $S_{n}$ is full. If so, send the contents of $S_{n}$ to CPE $n$ using RMA, and reset the sending buffer unit $S_{n}$.

- Step 3: Poll to process its own receiving buffer unit. If valid data is found in the receiving buffer unit, the reduction value, location, and other information of the receiving buffer unit will be resolved.

- Steps 4 and 5: If the original data of the reduction location are found to have been buffered into $b u f$, the update operation will be performed directly; otherwise, the buffered $b u f$ data will be flushed back to main memory first, and then the required data fragments will be buffered into buf with DMA.

This algorithm cleverly utilizes the idea of cache references to map the big data that are waiting for reduction into blocks in LDM, and then uses an RMA communication mechanism between CPEs to aggregate and batch the reduction information, so that the communication bandwidth between CPEs can be efficiently utilized. This method has been integrated into the Sunway-OpenACC. The performance of the calculation of the Hamiltonian has been greatly improved by using this method to solve the discrete reduction problem of the response Hamiltonian for large arrays.

\subsection{The explicit purification solver to get the response order density matrix}

The traditional method for obtaining the response density matrix involves solving the perturbation form of the Schrödinger equation (i.e., the Sternheimer equation) implicitly through a linear equation, which scales as $O\left(N^{3}\right)$ and presents a serious bottleneck to the calculations for large-scale systems. To reduce the 
scaling factor, the explicit purification method $[37,38]$ is a promising choice. Explicit purification converts the Sternheimer equation into a matrix-matrix multiplication problem involving only zero/first-order density and Hamiltonian matrices. Both density and Hamiltonian matrices can be sparse according to Kohn's nearsightedness principle [39], which is saying that, for a quantum mechanical system within an external potential, its local properties do not see a change of the external potential if the change is limited to a distant region. This is the fundamental principle behind almost all linear scaling algorithms[40, 41]. Here, we extend the usage of the second order trace-correcting purification (TC2) method to the response theory [38], which is called TC2-DFPT. Within TC2-DFPT, the response density matrix $P^{(1)}$ is explicitly constructed from the response Hamiltonian matrix $H^{(1)}$. As shown in Fig. 3(h), the response density matrix is calculated only with matrix-matrix multiplication, provides the base for computing the response density matrix explicitly and rapidly. The calculation of the parallel matrix multiplication is performed by minimizing the data communication between MPI tasks, the matrix is broadcasted and multiplied locally to compute a contribution to a local result matrix, and finally the result matrix is summed up. A detailed discussion of the local matrix multiplication will be present in Sec. 7.3.

\subsection{Mixed-precision computation}

The iterative DFT/DFPT solver gives us an opportunity to trade accuracy for performance. Within the inner purification cycle, the matrix-matrix multiplication is performed iteratively with double or half precision. Then, during the outer DFT/DFPT cycles, different levels of mixed precision can be performed with satisfactory stability and accuracy. In the mixed-half scheme, the half precision (16-bit) matrix-matrix multiplication in purification inner loop (response density matrix) is performed for the first several outer DFT/DFPT cycles, all other floating point operations (response density, response potential, response Hamiltonian) are conducted in double precision due to accuracy considerations. Then for the last several outer DFT/DFPT cycles, the double precision is used for all kernels to get the final converged results. We find the accuracy of the above mix-precision scheme gives absolute (relative) deviation of $1 \times 10^{-3} \mathrm{eV}\left(1 \times 10^{-4} \%\right)$ in the energy prediction and a absolute (relative) deviation of $2 \times 10^{-2} \mathrm{Bohr}^{3}(0.3 \%)$ in the DFPT prediction, which is acceptable for the engineering applications. The detailed discussion about the accuracy of the mixed-precision schemes is further validated in Sec.7.3.

\section{PERFORMANCE MEASUREMENT}

\subsection{HPC System and Environment}

The new-generation Sunway supercomputer is used for performance assessment in this work, which is the successor of the Sunway TaihuLight supercomputer. Similar to the Sunway TaihuLight system, the new Sunway supercomputer adopts a new generation of domestic high-performance heterogeneous many-core processors (SW26010Pro) and interconnection network chips in China. The system consists of computing system, interconnection system, software system, file system, peripheral service system, maintenance and diagnosis system, and cooling system. The software system is designed for the new application requirements such as scientific and engineering computing, AI, and big data, including basic software, parallel operating system, parallel language environment, parallel development environment, AI support software, application support system and other components. It supports tens of millions of core super-large-scale resource management and scheduling, provides system-wide monitoring management and fault tolerance mechanisms, and supports multi-level debugging and performance tuning of application software. For the SW26010Pro, the SACA (Sunway Accelerate Computing Architecture) library has been realized to support the dynamic loading mode and multilevel shared programming mechanism. The customized compiler supports $\mathrm{C} / \mathrm{C}++$, Fortran, it also supports parallel programming languages such as MPI (3.1), OpenMP and OpenACC and other parallel programming environments.

The SW26010Pro processor is designed for massive threads and data parallelism and to deliver high performance on parallel workloads. The peak performance of the SW26010Pro processor is 14.026 $\mathrm{TFLOP} / \mathrm{s}$ in double precision and 55.296 TFLOP/s in half precision, the aggregated memory bandwidth of $307.2 \mathrm{~GB} / \mathrm{s}$. The architecture of the SW26010Pro processor is shown in Fig. 4. Each processor contains 6 core-groups (CGs), with 65 cores in each CG, and in total 390 cores. Each CG contains one management processing element (MPE), one cluster of computing processing elements (CPEs) and one memory controller (MC). The MPE (operates at a clock frequency of $2.1 \mathrm{GHz}$ ) within each CG is used for computations, management and communication. The CPEs (operates at a clock frequency of $2.25 \mathrm{GHz}$ ) is organized as an $8 \times 8$ mesh ( 64 cores) and is designed to maximize the aggregated computing capability and to minimize the complexity of the micro-architecture.

Each SW26010Pro processor contains 96 GB memory, with 16 GB memory in each CG. The MPE and the CPEs within the same CG share the same memory which is controlled by the MC. Each CPE has a $32 \mathrm{~KB}$ L1 instruction cache, and a $256 \mathrm{~KB}$ scratch pad memory (SPM, also called the Local Data Memory (LDM)), which serves the same function as the $\mathrm{L} 1$ cache. The data storage space can also be configured as a local data cache (LDCache) which is automatically managed by the hardware. Data transfer between LDM and main memory can be realized by direct memory access (DMA), and data transfer between LDCache and main memory can also be realized by conventional load/storage instructions. The CPE adopts the SW64 instructions and provide 512-bit SIMD support. All the double precision, single precision, half precision floatingpoint computation and integer computation are supported. The CPE cluster is organized with a mesh network to achieve low-latency data communication ( $\mathrm{P} 2 \mathrm{P}$ and collective communications) among the CPEs in one CG, which is called remote scratch pad memory access (RMA). For the network, within the processor, the 6 CGs are connected using a network on chip (NoC). The 256 processors inside a supernode are fully connected through a customized network board, then the supernodes are connected with the central network switches.

\subsection{Physical Systems}

To measure the performance of the Raman spectra calculations with FHI-aims, we considered the receptor binding domain (RBD) of the surface Spike glycoprotein (S protein) in SARS-CoV-2. SARS-CoV-2 


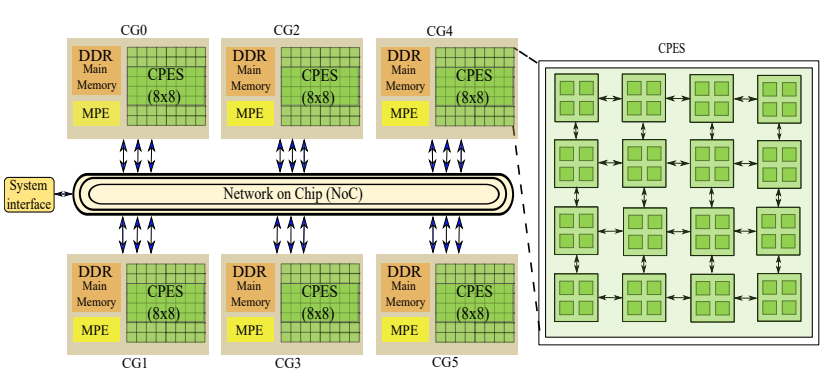

Figure 4: The architecture of the SW26010Pro processor.

is the novel coronavirus behind the COVID-19 pandemic, which has spread rapidly around the world over the past 18 months. The RBD within the surface $S$ protein engages the human angiotensinconverting enzyme 2 (ACE2) receptor. To design small-molecule antiviral drugs/inhibitors to prevent the further spread of COVID19 , one promising therapeutic strategy is to design high-affinity inhibitors of the SARS-CoV-2 S protein RBD to compete with ACE2 binding. Structural information about the RBD will provide a fundamental understanding about the interaction between atoms. The RBD protein complex contains 3006 atoms. All calculations used light settings and the LDA functional in FHI-aims.

\subsection{Measurement Methodology}

For the Raman spectra calculation of large biological systems, there are four computation kernels as shown in Fig. 2. Of these, the calculation of response density matrix $P^{(1)}$ comprises $90 \%$ of the FLOP count. Thus, the major computation kernel is for the evaluation of the first-order density matrix, which involves matrixmatrix multiplication. The FLOP count of each DFT-SCF iteration is $\left(N_{\mathrm{TC} 2-\text { iterations }}+4\right) \times\left(2 \times N_{\text {basis }}^{3}+3 \times N_{\text {basis }}^{2}\right)$. Here, $N_{\mathrm{TC} 2 \text {-iterations }}$ refers to the total number of purification cycles, $N_{\text {basis }}$ refers to the dimension of the (response) density matrix, there are four additional matrix-matrix multiplications because of the orthogonal transformation and permutation operations, and $3 \times N_{\text {basis }}^{2}$ refers to the increment and dot product operations within each TC2 iteration. The overall FLOP count for each DFPT-SCF iteration is three times that of the DFT part since the number of the matrixmatrix multiplication for the response density matrix calculation is tripled [42], so the overall FLOP count of each DFPT-CPSCF iteration is $\left[\left(N_{\mathrm{TC} 2-\text { iterations }}+4\right) \times\left(2 \times N_{\text {basis }}^{3}+3 \times N_{\text {basis }}^{2}\right)\right] \times 3$.

\section{PERFORMANCE RESULTS}

\subsection{Simulation Validation}

The dielectric constant is a measure of the amount of electric potential energy, in the form of the induced polarization stored in a given volume of material under the action of an electric field. It is directly related to the polarizability,

$$
\varepsilon_{\gamma \delta}^{\infty}=\delta_{\gamma \delta}+\frac{4 \pi}{V_{u c}} \alpha_{\gamma \delta},
$$

where $V_{u c}$ refers to the volume of the unit cell.

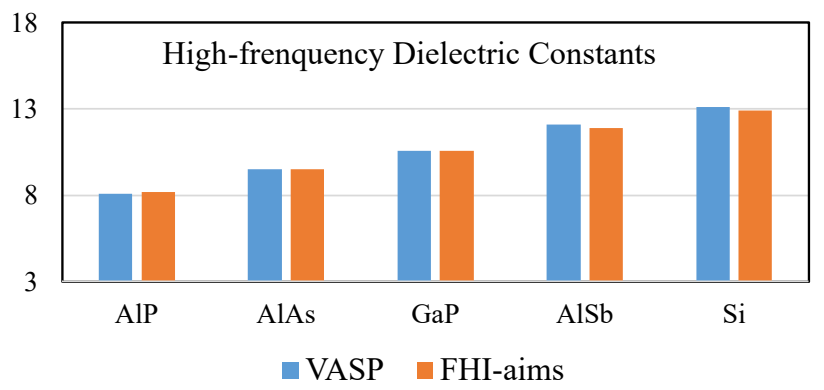

Figure 5: High-frequency dielectric constants of various semiconductors computed at the PBE level, comparison between our all-electron approach and the VASP result for five materials.

To validate our all-electron approach, we have calculated the dielectric constant of several semiconductors using the generalized gradient approximation (GGA-PBE [43]) and compared it with theoretical data from a plane wave (PW) code ( VASP). The all-electron calculations have been performed at the theoretical equilibrium lattice constant using $16 \times 16 \times 16 \mathrm{k}$-points in the primitive unit cell and "tight" basis set and integration settings. The VASP results were obtained using the PW basis set and the projector augmented wave method (PAW) [44]. Fig. 5 illustrates the excellent agreement between our all-electron full-potential (FHI-aims) approach and the PW-PAW (VASP) methods, the relative errors are within $1 \%$.

We also compared the response density of the RBD protein on Tianhe-2 and Sunway, the mean absolute error (MAE) for the difference between the response density obtained via Sunway and Tianhe- 2 is $1 \times 10^{-9} e / \AA^{3}$, our results on the above two platforms are in good agreement within machine precision.

\subsection{Speedup}

We use the calculation of the response potential as an example to illustrate the performance improvement for different optimization methods on one new Sunway node, which contains 390 cores (6 MPEs with $384 \mathrm{CPEs})$. Fig. 6 compares different versions optimized by a series of methods. We used different settings (as shown in Fig. 6) to calculate the Raman spectrum of solid silicon with various numbers of grid and basis sets. First, the DMA loop tiling method yielded performance improvements ranging from $10-15 \times$ by increasing the data locality. Second, double buffering was introduced after loop tiling to overlap the data fetching to SPM and the computation. The performance results show that when double buffering is enabled, all the data fetching operations can be completely overlapped with the computation, further accelerating the performance to $16 \times$. Third, we applied vectorization and kernel fusion to further improve the instruction level parallelism, resulting in an overall performance speedup of around $30 \times$ compared with the original MPE version.

Fig. 7 presents an evaluation of the performance improvements over CPU calculations performed on Tianhe-2 with an Intel CPU. We used an RBD protein containing 3006 atoms as the calculation target. Compared with the CPU implementation, the innovations 


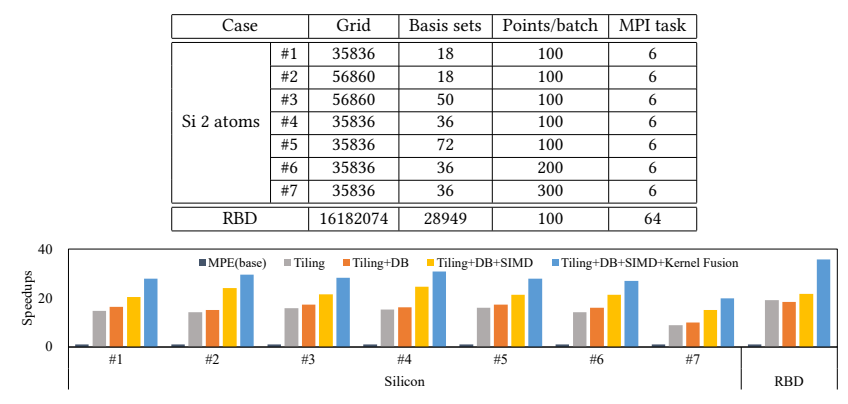

Figure 6: The performance comparison among different optimization steps in the response potential calculation.

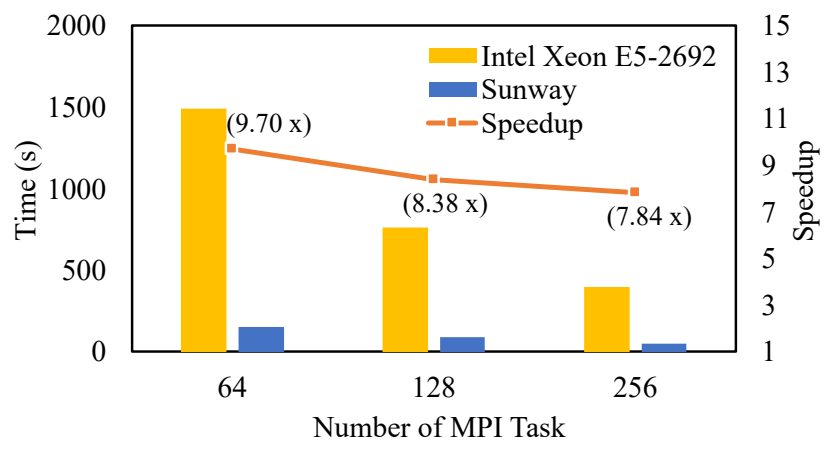

Figure 7: Performance comparison of the total time per DFPT iteration for RBD protein containing 3006 atoms.

described in Sec. 5 produced an overall speedup in the DFPT total time per cycle ranging from $9.7 \times$ with 64 processes to $7.8 \times$ with 256 processes in the strong scaling test.

\subsection{Local matrix calculation and mix-precision}

During the response density matrix calculation, the local matrix multiplication need to be performed. In order to minimize the commutation volume, the local matrix is gathered in the sparse matrix form. To perform local matrix multiplication operation, one can either use sparse matrix multiplication (sparse) or use sparse-todense conversion followed by dense multiplication and then densto-sparse conversion (trans). We found there is a trade-off between memory resources and computing capability. The 'trans' method has high memory access bandwidth (bandwidth utilization is close to $90 \%$.) and can make better use of the vectorization function in SW26010Pro, while the 'sparse' method have a large number of discrete memory access operations and are not suitable for vectorization. So the 'trans' method performs better than the 'sparse' method in our study case.

The accuracy of the mixed precision models is investigated by comparing the results of the total energy and polarizability calculated with double-precision. We take water molecule as an example since it is a benchmark test case in the DFT/DFPT calculation, we perform the half-precision calculation in the density matrix (response density matrix) part in the first 25(40) DFT(DFPT) cycles, and then change back to double-precision calculation for the last few
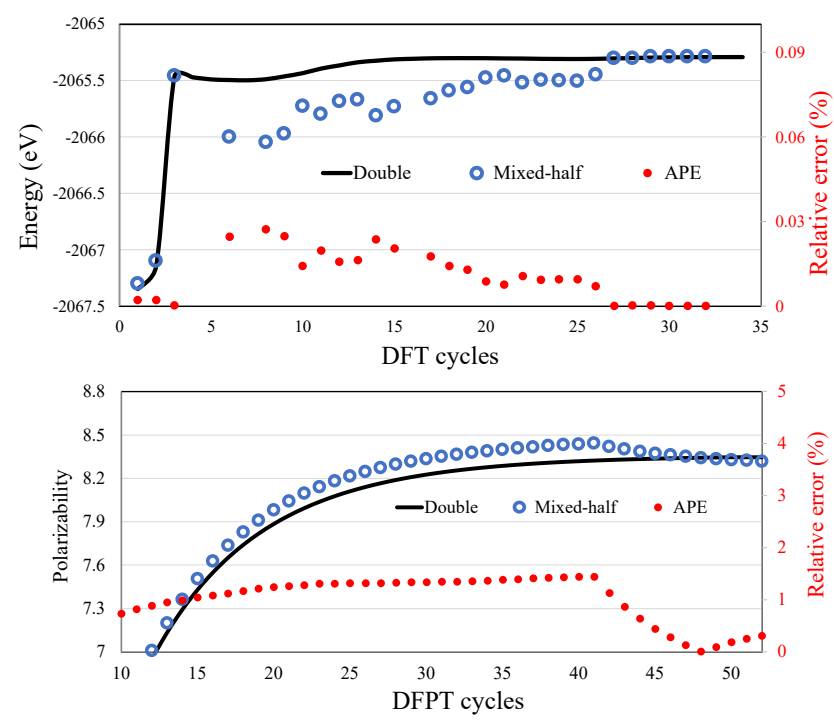

Figure 8: Comparison of double-precision and halfprecision in the DFT and DFPT calculation. Additionally, the absolute percentage errors (APE) with respect to double-precision calculations are given.

DFT(DFPT) cycles, as shown in Fig. 8, the accuracy of our iterationmix-half scheme has the absolute (relative) error of $1 \times 10^{-3} \mathrm{eV}$ $\left(1 \times 10^{-4} \%\right)$ in the energy prediction, and has the absolute (relative) deviation of $2 \times 10^{-2} \mathrm{Bohr}^{3}(0.3 \%)$ in the DFPT prediction, which is enough for the accurate prediction of physical observables. Therefore, the mix-precision methods can be used for the calculation of the physical observables.

\subsection{Scalability Results}

Fig. 9 shows the strong scaling performance of the Raman spectra calculations for the RBD protein complex. In all calculations, each MPI sub-group was mapped to 256 processes, and the total number of polarizabilities was set to 1175 for the strong scaling calculation. The code shows good strong scaling performance: as the number of Sunway processes increases from 10,240 to 300,800 for the calculation (number of cores increases from 665,600 to $19,552,000$ ), the parallel efficiency exceeds $80 \%$ and $25 \times$ speedup.

Fig. 10 shows the weak scaling of the Raman spectra calculation for the RBD protein. To obtain the weak scaling data, we timed both the DFT and DFPT calculations on the RBD protein complex with an increasing number of polarizabilities (256 processes per polarizability). The parallel algorithm in the Raman spectra calculation weakly scales with the number of the polarizabilities, and the calculation achieves good weak scaling as the number of cores grows.

Furthermore, in order to examine the largest system that the current DFPT code can simulate, we perform the DFPT calculation to get the polarizability with polyethylene molecular system $(\mathrm{H}[\mathrm{C} 2 \mathrm{H} 4] \mathrm{nH})$, and the largest system we can simulate containing 500,000 atoms with the adaptive load balancing method. 


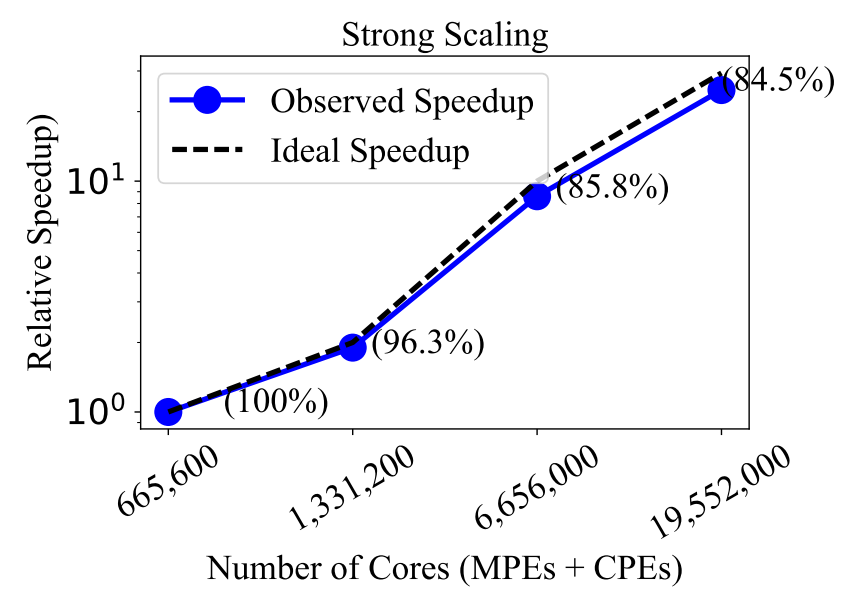

Figure 9: Strong scalability of the Raman spectra computation time for an RBD complex containing 3006 atoms. The blue line is the relative speedup and the black dash line is the ideal speedup. Parallel efficiency values are annotated in parentheses, respectively.

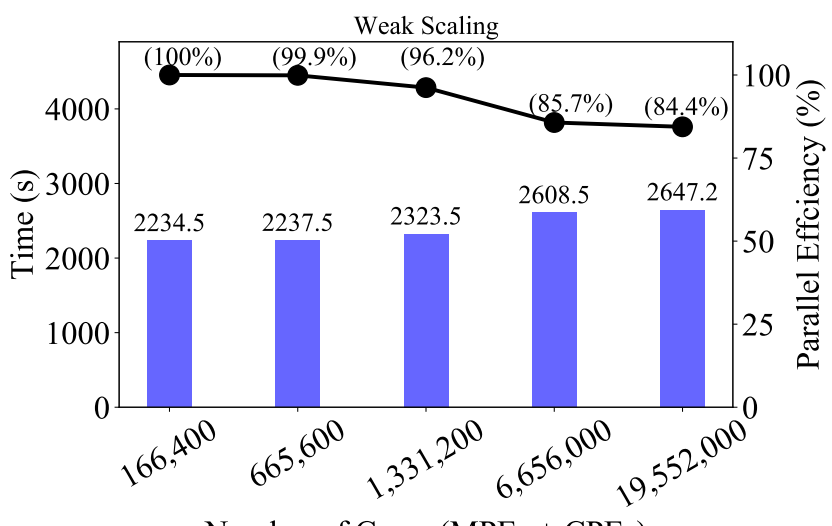

Number of Cores (MPEs + CPEs)

Figure 10: Weak scalability of the Raman spectra computation time for an the RBD complex containing 3006 atoms. Here, the number of the polarizabilities in the Raman spectra calculation increases with the number of the cores (MPEs + CPEs). The blue bars denote the simulation time, the black line is the weak parallel efficiency. Simulation time and parallel efficiency values are annotated on the top of the bars and in parentheses respectively.

\subsection{Peak Performance}

We use the Raman spectra simulation of RBD protein system to measure the throughput performance of the most computationally demanding kernel (response density matrix). The results are shown in Fig. 11. In this experiment, the computational cost was around 489 PFLOP per purification iteration within the response density matrix calculation. The performance of $468.5 \mathrm{PFLOP} / \mathrm{s}$ is achieved in double precision, and $813.7 \mathrm{PFLOP} / \mathrm{s}$ in mixed half precision with 625,920 processes (40,684,800 cores), which has included communication.

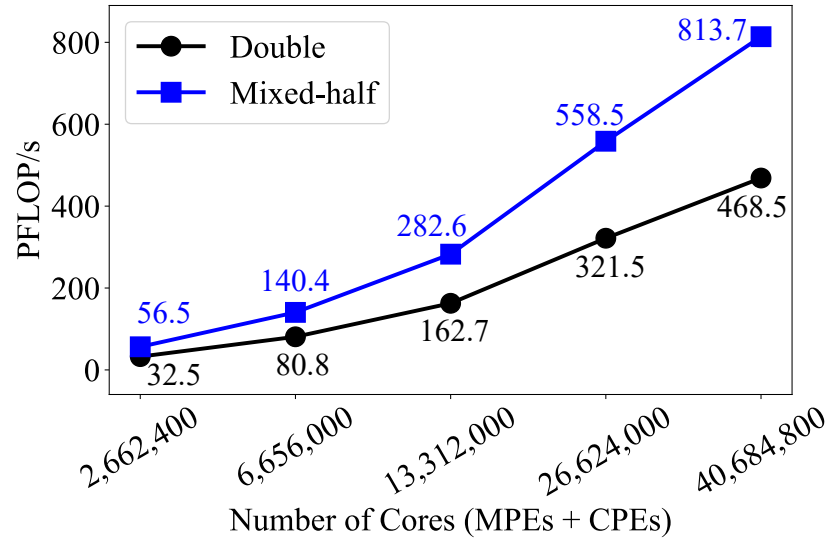

Figure 11: The performance (PFLOP/s) of the Raman spectra simulation on the new-generation Sunway supercomputer.

We also use the Roofline model to analyze the bottlenecks of our Raman simulation. We find the kernel of the response density matrix is compute-bound in double-precision, but is memory-bound in halfprecision. The communication within the response density matrix calculation is the allgather collective, and has been overlapped with computation.

\section{IMPLICATIONS}

This section discusses the application of DFPT to a large realistic biological system. Raman spectroscopy is increasingly being applied to biological systems [45], because it can provide chemical and composition information for proteins in essentially all physical states, and can probe the structural changes in proteins that result from protein-ligand interactions. Using the DFPT method, the Raman spectra can be calculated through harmonic approximation.

The computations are based on the crystal structure of the RBD protein reported by Wang et al. [46] at a resolution of $2.5 \AA$; the protein data bank (PDB) file number of this protein is 6LZG. The hydrogen atoms of the protein were added using AmberTools.

It has been reported that ACE2 is the cellular receptor for the SARS-CoV-2 coronavirus, with the RBD of the surface $S$ protein used to engage ACE2. One promising therapeutic strategy is to design high-affinity inhibitors for the SARS-CoV-2 S protein RBD to compete with ACE2 binding. The Raman spectra of the RBD may provide the necessary structural information. The Raman spectra for the RBD protein were calculated with the LDA functional and a "light" basis set. The Raman spectra and a comparison with experimental data are shown in Fig. 12. The smearing of the theoretical Raman spectra was set to $5 \mathrm{~cm}^{-1}$, except for the spectra within the amide III zone, which were not smeared in order to illustrate finer vibrational information.

Overall, there is good agreement between the calculated and experimental Raman spectra [47]. Although the intensities have relatively small differences, characteristic patterns are easily visible. The S-S bond stretching bands are located in the $500-550 \mathrm{~cm}^{-1}$ region, which shows that there are S-S bridges in the protein. These 


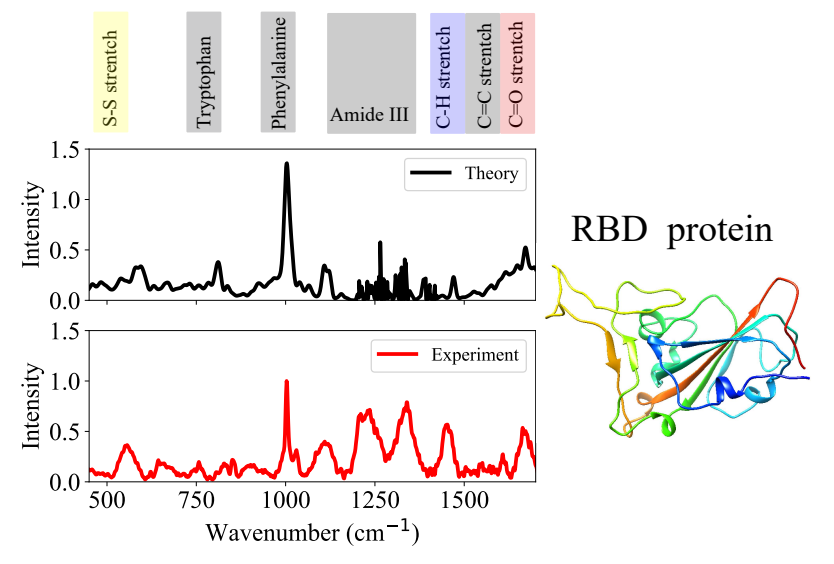

Figure 12: Simulated and experimental Raman spectra of the RBD protein, demonstrating the sensitivity power of the Raman spectra in probing the protein structure.

bands were observed in both the experimental and calculated spectra. The bands near $800 \mathrm{~cm}^{-1}$ observed in the experimental and theoretical spectra are the aromatic amino-acid (Tyrosine) bands, which come from the in-plane breathing mode of the phenol ring. The Trp/Phe aromatic Raman signal around $1001 \mathrm{~cm}^{-1}$ and the Trp band at $1112 \mathrm{~cm}^{-1}$ (calculated at 1003 and $1117 \mathrm{~cm}^{-1}$, respectively) are related to the breathing mode of the Phenylalanine. These distinct spectral features have been reproduced well in the simulation. The amide III spectral region (coupled $\mathrm{C}-\mathrm{N}$ stretching and $\mathrm{N}-\mathrm{H}$ bending vibrations, around $1200-1360 \mathrm{~cm}^{-1}$ ) can be correlated with the amide I band (stretching vibration of $\mathrm{C}=\mathrm{O}$, around $1650 \mathrm{~cm}^{-1}$ ) to obtain some additional details about amide I. The two bands in this amide III region have a higher intensity in the experimental measurement than in our calculation, as does the $\mathrm{C}=\mathrm{C}$ stretching band. The remaining part of the experimental spectrum, with bands at around $1650 \mathrm{~cm}^{-1}$, was also obtained in our calculation, and we obtained a similar relative intensity of the band at $1650 \mathrm{~cm}^{-1}$ for the amide I band compared with the measured spectrum.

Finally, we used the simulated Raman spectra to provide direct evidence of the structural changes caused by the ligand-protein interactions. Fig. 13 shows the Raman spectra of the HIV-1 protease complex with a tripeptide inhibitor (PDB file number 1a30). We can see a significant change in the characteristic bands when the ligand is placed into the pocket, which indicates the presence of an interaction between the protein and the ligand. By investigating the shifts in these peaks experimentally and theoretically, the structural changes of the protein-ligand complex can be tracked. Looking at the calculated Raman spectra in Fig. 13, we can see two bands at around $960 \mathrm{~cm}^{-1}$ and $1038 \mathrm{~cm}^{-1}$ in the middle panel (pocket without the ligand). These are related to the breathing mode of phenylalanine and to $\mathrm{C}-\mathrm{C}$ and $\mathrm{C}-\mathrm{N}$ stretching vibrations. However, once the ligand is inserted into the pocket, bonding occurs and these stretching modes become weaker, as can be seen from the lower panel. The bands around $1230-1300 \mathrm{~cm}^{-1}$ in the spectrum of the protein pocket are related to amide III and are associated with coupled $\mathrm{C}-\mathrm{N}$ stretching and $\mathrm{N}-\mathrm{H}$ bending vibrations of the peptide group. Such bands become flatter and weaker when the ligand is in

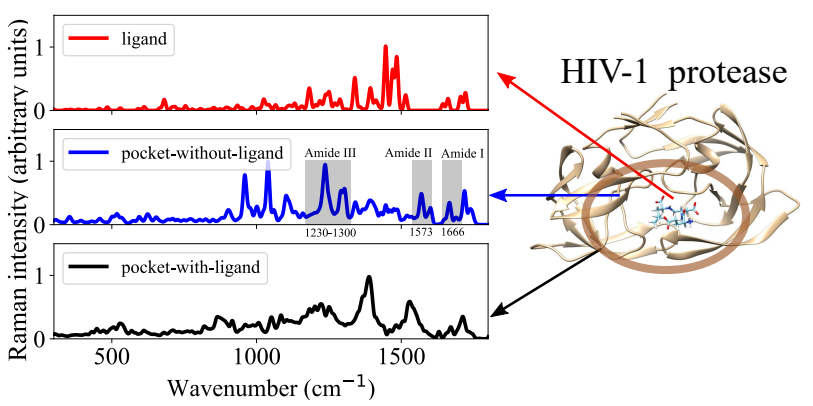

Figure 13: Raman spectra of an HIV-1 protease complex with a tripeptide inhibitor. The upper, middle, and lower panels show the Raman spectra of the ligand that of the protein pocket without the ligand, and that of the whole pocket with the ligand, respectively.

the pocket, which is a result of the structural variation. Similarly, the amide II band at around $1573 \mathrm{~cm}^{-1}$ and the amide I band related to the stretching vibration of $\mathrm{C}=\mathrm{O}$ at $1666 \mathrm{~cm}^{-1}$ change accordingly. Thus, using Raman spectroscopy, we have obtained direct evidence of structural changes, and we can use this approach to determine whether the ligand has been combined with the protein.

The innovations realized in this study demonstrate that the $a b$ initio simulation of Raman spectra in FHI-aims is suitable for use in future exascale machines. Three levels of parallelization have been implemented according to the nature of the physical problem and the many-core architecture. To the best of our knowledge, this is the first reported quantum perturbation calculation for Raman spectra with as many as 3006 atoms and that has been scaled to around ten million cores. Furthermore, the ability to conduct Raman spectra simulations of realistic biological systems has been demonstrated, suggesting new applications of the QM approach to biological systems. This is the starting point for using the QM method to probe the structural changes in proteins that result from protein-ligand interactions, opening up the possibility of improved accuracy in virtual drug screening. We can further use our QM tool to investigate the biochemical dynamic processes, so that we will be not just scratching the surface of the understanding of the proteins, but to gain a more thorough understanding of the real biological systems.

\section{ACKNOWLEDGMENT}

This work was supported by the National Natural Science Foundation of China (grant no. 22003073), State Key Laboratory of Computer Architecture Foundation under Grant No. CARCH 4205 and $\mathrm{CARCH} 4411$. The authors would like to thank Prof. Jinlong Yang, Prof. Wanzhen Liang, Prof. Yi Luo, Prof. Matthias Scheffler for inspiring discussions.

\section{REFERENCES}

[1] V. Blum, R. Gehrke, F. Hanke, P. Havu, V. Havu, X. Ren, K. Reuter, and M. Scheffler, "Ab initio molecular simulations with numeric atom-centered orbitals," Comput. Phys. Commun., vol. 180, no. 11, pp. 2175-2196, Nov. 2009. [Online]. Available: http://linkinghub.elsevier.com/retrieve/pii/S0010465509002033

[2] F. Gygi, C. W. Ueberhuber, J. Lorenz, E. W. Draeger, M. Schulz, B. R. de Supinski, J. A. Gunnels, V. Austel, J. C. Sexton, F. Franchetti, and S. Kral, "Gordon Bell 
finalists I-Large-scale electronic structure calculations of high- $Z$ metals on the BlueGene/L platform," in Proceedings of the 2006 ACM/IEEE conference on Supercomputing - SC '06. New York, New York, USA: ACM Press, 2006, p. 45 [Online]. Available: http://portal.acm.org/citation.cfm?doid=1188455.1188502

[3] Y. Hasegawa, M. Kurokawa, H. Inoue, I. Miyoshi, M. Yokokawa, J.-I. Iwata, M. Tsuji, D. Takahashi, A. Oshiyama, K. Minami, T. Boku, F. Shoji, and A. Uno, "First-principles calculations of electron states of a silicon nanowire with 100,000 atoms on the K computer," in Proceedings of 2011 International Conference for High Performance Computing, Networking, Storage and Analysis on - SC '11. New York, New York, USA: ACM Press, 2011, p. 1. [Online]. Available: http://dl.acm.org/citation.cfm?doid=2063384.2063386

[4] J. L. Fattebert, D. Osei-Kuffuor, E. W. Draeger, T. Ogitsu, and W. D. Krauss, "Modeling Dilute Solutions Using First-Principles Molecular Dynamics: Computing more than a Million Atoms with over a Million Cores," in International Conference for High Performance Computing, Networking, Storage and Analysis, SC, no. November, 2016, pp. 12-22.

[5] S. Das, P. Motamarri, V. Gavini, B. Turcksin, Y. W. Li, and B. Leback, "Fast, scalable and accurate finite-element based ab initio calculations using mixed precision computing," in Proceedings of the International Conference for High Performance Computing, Networking, Storage and Analysis. New York, NY, USA: ACM, nov 2019, pp. 1-11. [Online]. Available: https://dl.acm.org/doi/10.1145/3295500.3357157

[6] J. VandeVondele, U. Borštnik, and J. Hutter, "Linear Scaling Self-Consistent Field Calculations with Millions of Atoms in the Condensed Phase," fournal of Chemical Theory and Computation, vol. 8, no. 10, pp. 3565-3573, oct 2012 [Online]. Available: https://pubs.acs.org/doi/10.1021/ct200897x

[7] A. N. Ziogas, T. Ben-Nun, G. I. Fernández, T. Schneider, M. Luisier, and T. Hoefler "A data-centric approach to extreme-scale ab initio dissipative quantum transport simulations," in International Conference for High Performance Computing Networking, Storage and Analysis, SC, 2019.

[8] A. Nakata, J. S. Baker, S. Y. Mujahed, J. T. L. Poulton, S. Arapan, J. Lin, Z. Raza, S. Yadav, L. Truflandier, T. Miyazaki, and D. R. Bowler, "Large scale and linear scaling DFT with the CONOUEST code," The fournal of Chemical Physics, vol. 152, no. 16, p. 164112, apr 2020. [Online]. Available: https://doi.org/10.1063/5.0005074http://aip.scitation.org/doi/10.1063/5.0005074

[9] J. C. Prentice, J. Aarons, J. C. Womack, A. E. Allen, L. Andrinopoulos, L. Anton, R. A. Bell, A. Bhandari, G. A. Bramley, R. J. Charlton, R. J. Clements, D. J. Cole, G. Constantinescu, F. Corsetti, S. M. Dubois, K. K. Duff, J. M. Escartín, A. Greco, Q. Hill, L. P. Lee, E. Linscott, D. D. O’Regan, M. J. Phipps, L. E. Ratcliff, Á. R Serrano, E. W. Tait, G. Teobaldi, V. Vitale, N. Yeung, T. J. Zuehlsdorff, J. Dziedzic, P. D. Haynes, N. D. Hine, A. A. Mostofi, M. C. Payne, and C. K. Skylaris, "The ONETEP linear-scaling density functional theory program," fournal of Chemical Physics, vol. 152, no. 17, 2020

[10] L. E. Ratcliff, W. Dawson, G. Fisicaro, D. Caliste, S. Mohr, A. Degomme, B. Videau, V. Cristiglio, M. Stella, M. D’Alessandro, S. Goedecker, T. Nakajima, T. Deutsch, and L. Genovese, "Flexibilities of wavelets as a computational basis set for large-scale electronic structure calculations," The fournal of chemical physics, vol. 152, no. 19, p. 194110, 2020. [Online]. Available: https://doi.org/10.1063/5.0004792

[11] P. Giannozzi, S. Baroni, N. Bonini, M. Calandra, R. Car, C. Cavazzoni, D. Ceresoli, G. L. Chiarotti, M. Cococcioni, I. Dabo, A. Dal Corso, S. de Gironcoli, S. Fabris, G. Fratesi, R. Gebauer, U. Gerstmann, C. Gougoussis, A. Kokalj, M. Lazzeri, L. Martin-Samos, N. Marzari, F. Mauri, R. Mazzarello, S. Paolini, A. Pasquarello, L. Paulatto, C. Sbraccia, S. Scandolo, G. Sclauzero, A. P. Seitsonen, A. Smogunov, P. Umari, and R. M. Wentzcovitch, "OUANTUM ESPRESSO: a modular and open-source software project for quantum simulations of materials," fournal of Physics: Condensed Matter, vol. 21, no. 39, p. 395502, sep 2009. [Online]. Available: https://iopscience.iop.org/article/10.1088/0953-8984/21/39/395502

[12] V. W. Elloh, A. Yaya, A. K. Mishra, G. Gebreyesus, K. Kan-Dapaah, E. K. K. Abavare, and B. Onwona-Agyeman, "Computational modelling of structural, electronic, optical and vibrational properties of $\mathrm{pvk} / \mathrm{c}-60$ nanoheterostructure interfaces," Biointerface Research in Applied Chemistry, vol. 11, no. 3, pp. 10 864-10 884, 2021. [Online]. Available: <GotoISI>://WOS:000591668100085

[13] R. e. a. Dovesi, "The CRYSTAL code, 1976-2020 and beyond, a long story," The fournal of chemical physics, vol. 152, no. 20, p. 204111, 2020. [Online]. Available: https://doi.org/10.1063/5.0004892

[14] S. Salustro, A. M. Ferrari, R. Orlando, and R. Dovesi, "Comparison between cluster and supercell approaches: the case of defects in diamond," Theoretical Chemistry Accounts, vol. 136, no. 4, pp. 1-13, 2017.

[15] K. Reiter, F. Weigend, L. N. Wirz, M. Dimitrova, and D. Sundholm, "Magnetically Induced Current Densities in Toroidal Carbon Nanotubes," Journal of Physical Chemistry C, vol. 123, no. 24, pp. $15354-15365,2019$.

[16] S. Luber and M. Reiher, "Theoretical raman optical activity study of the $\beta$ domain of rat metallothionein," Journal of Physical Chemistry B, vol. 114, no. 2, pp. 1057$1063,2010$.

[17] H. Shang, C. Carbogno, P. Rinke, and M. Scheffler, "Lattice dynamics calculations based on density-functional perturbation theory in real space," Computer Physics Communications, vol. 215, pp. 26-46, jun 2017. [Online]. Available: http://www.sciencedirect.com/science/article/pii/S0010465517300437https:
//linkinghub.elsevier.com/retrieve/pii/S0010465517300437

[18] H. Shang, N. Raimbault, P. Rinke, M. Scheffler, M. Rossi, and C. Carbogno, "All-electron, real-space perturbation theory for homogeneous electric fields: theory, implementation, and application within DFT," New fournal of Physics, vol. 20, no. 7, p. 073040, jul 2018. [Online]. Available: http://stacks.iop.org/ $1367-2630 / 20 / \mathrm{i}=7 / \mathrm{a}=073040$ ?key=crossref.b45b8680fc0308226fe0611417a68450

[19] P. G. et al., "Quantum espresso: a modular and open-source software project for quantum simulations of materials," fournal of Physics: Condensed Matter, vol. 21, no. 39, p. 395502 (19pp), 2009. [Online]. Available: http: //www.quantum-espresso.org

[20] G. Kresse and J. Hafner, "Ab initio molecular dynamics for liquid metals," Phys. Rev. B, vol. 47, pp. 558-561, Jan 1993. [Online]. Available: https: //link.aps.org/doi/10.1103/PhysRevB.47.558

[21] A. H. e. a. Romero, "ABINIT: Overview and focus on selected capabilities," Journal of Chemical Physics, vol. 152, no. 12, 2020. [Online]. Available: https://doi.org/10.1063/1.5144261

[22] X. Andrade, D. Strubbe, U. De Giovannini, A. H. Larsen, M. J. T. Oliveira, J. Alberdi-Rodriguez, A. Varas, I. Theophilou, N. Helbig, M. J. Verstraete, L. Stella, F. Nogueira, A. Aspuru-Guzik, A. Castro, M. A. L. Marques, and A. Rubio, "Realspace grids and the octopus code as tools for the development of new simulation approaches for electronic systems," Phys. Chem. Chem. Phys., vol. 17, pp. 31 371-31 396, 2015. [Online]. Available: http://dx.doi.org/10.1039/C5CP00351B

[23] T. D. e. a. Kühne, "CP2K: An electronic structure and molecular dynamics software package -Quickstep: Efficient and accurate electronic structure calculations," Journal of Chemical Physics, vol. 152, no. 19, 2020. [Online]. Available: https://doi.org/10.1063/5.0007045

[24] K. e. a. Lejaeghere, "Reproducibility in density functional theory calculations of solids," Science, vol. 351, no. 6280, pp. aad3000-aad3000, mar 2016. [Online]. Available: http://science.sciencemag.org/content/351/6280/aad3000.abstracthttp: //www.sciencemag.org/cgi/doi/10.1126/science.aad3000

[25] M. Frisch, M. Head-Gordon, and J. Pople, "Direct analytic scf second derivatives and electric field properties," Chem. Phys., vol. 141, no. 2-3, pp. $189-$ 196, 1990. [Online]. Available: http://www.sciencedirect.com/science/article/pii/ 030101049087055G

[26] B. Delley, "An all-electron numerical method for solving the local density functional for polyatomic molecules," f. Chem. Phys., vol. 92, no. 1, p. 508, 1990. [Online]. Available: http://scitation.aip.org/content/aip/journal/jcp/92/1/10.1063/ 1.458452

[27] P. Bouř, J. Sopková, L. Bednárová, P. Maloň, and T. A. Keiderling, “Transfer of molecular property tensors in cartesian coordinates: A new algorithm for simulation of vibrational spectra," Journal of Computational Chemistry, vol. 18, no. 5, pp. 646-659, apr 1997. [Online]. Available: https://doi.org/10.1002/(SICI) 1096-987X(19970415)18:5\{\%\}3C646::AID-JCC6\{\%\}3E3.0.COhttp://2-n

[28] F. Pascale, S. Salustro, A. Maria, F. Michel, R. Philippe, and D. A. Roberto, "The Infrared spectrum of very large ( periodic) systems : global versus fragment strategies - the case of three defects in diamond," Theoretical Chemistry Accounts, vol. 137, no. 12, pp. 1-7, 2018. [Online]. Available: https://doi.org/10.1007/s00214-018-2380-3

[29] N. S. Bieler, M. P. Haag, C. R. Jacob, and M. Reiher, "Analysis of the Cartesian Tensor Transfer Method for Calculating Vibrational Spectra of Polypeptides," Journal of Chemical Theory and Computation, vol. 7, no. 6, pp. 1867-1881, jun 2011. [Online]. Available: https://doi.org/10.1021/ct2001478

[30] S. Yamamoto, X. Li, K. Ruud, and P. Bouř, "Transferability of Various Molecular Property Tensors in Vibrational Spectroscopy," fournal of Chemical Theory and Computation, vol. 8, no. 3, pp. 977-985, mar 2012. [Online]. Available: https://doi.org/10.1021/ct200714h

[31] A. D. Becke, "A multicenter numerical integration scheme for polyatomic molecules," f. Chem. Phys., vol. 88, no. 4, pp. 2547-2553, 1988. [Online]. Available: http://scitation.aip.org/content/aip/journal/jcp/88/4/10.1063/1.454033

[32] V. Havu, V. Blum, P. Havu, and M. Scheffler, "Efficient integration for all-electron electronic structure calculation using numeric basis functions," F. Comput. Phys., vol. 228, no. 22, pp. 8367-8379, Dec. 2009. [Online]. Available: http://linkinghub.elsevier.com/retrieve/pii/S0021999109004458

[33] M. S. Lam and M. Wolf, "A data locality optimizing algorithm," in Proceedings of the ACM SIGPLAN 1991 Conference on Programming Language Design and Implementation, ser. PLDI 91. New York, NY, USA: ACM, 1991, pp. 30-44.

[34] S. Coleman and K. S. McKinley, "Tile size selection using cache organization and data layout," in Proceedings of the ACM SIGPLAN 1995 Conference on Programming Language Design and Implementation, ser. PLDI 95. New York, NY, USA: ACM, 1995, pp. 279-290.

[35] W. Zhao, H. Fu, J. Fang, W. Zheng, L. Gan, and G. Yang, "Optimizing convolutional neural networks on the sunway taihulight supercomputer," ACM Transactions on Architecture and Code Optimization (TACO), vol. 15, no. 1, pp. 1-26, 2018.

[36] B. Khailany and B. Khailany, "Cudadma: Optimizing gpu memory bandwidth via warp specialization," in High Performance Computing, Networking, Storage and Analysis, 2011.

[37] A. M. N. Niklasson, "Expansion algorithm for the density matrix," Phys. Rev. B, vol. 66, p. 155115, Oct 2002. [Online]. Available: https://link-aps-org-443.webvpn. 
las.ac.cn/doi/10.1103/PhysRevB.66.155115

[38] A. M. N. Niklasson and M. Challacombe, "Density Matrix Perturbation Theory," Physical Review Letters, vol. 92, no. 19, p. 193001, may 2004. [Online]. Available: https://ink.aps.org/doi/10.1103/PhysRevLett.92.193001

[39] W. Kohn, "Density functional and density matrix method scaling linearly with the number of atoms," Phys. Rev. Lett., vol. 76, pp. 3168-3171, Apr 1996. [Online] Available: https://link.aps.org/doi/10.1103/PhysRevLett.76.3168

[40] D. R. Bowler and T. Miyazaki, "Calculations for millions of atoms with density functional theory: linear scaling shows its potential," $\mathcal{F}$. Phys Condens. Matter, vol. 22, no. 7, p. 74207, 2010. [Online]. Available: http //stacks.iop.org/0953-8984/22/i=7/a=074207

[41] H. Shang, H. Xiang, Z. Li, and J. Yang, "Linear scaling electronic structure calculations with numerical atomic basis set," International Reviews in Physical Chemistry, vol. 29, no. 4, pp. 665-691, oct 2010. [Online]. Available: http://www.tandfonline.com/doi/abs/10.1080/0144235X.2010.520454

[42] H. Shang, W. Liang, Y. Zhang, and J. Yang, "Efficient parallel linear scaling method to get the response density matrix in all-electron real-space density-functional perturbation theory," Computer Physics Communications, vol. 258, p. 107613 2021. [Online]. Available: https://doi.org/10.1016/j.cpc.2020.107613

[43] J. P. Perdew, K. Burke, and M. Ernzerhof, "Generalized gradient approximation made simple," Phys. Rev. Lett., vol. 77, pp. 3865-3868, Oct 1996. [Online]
Available: https://link.aps.org/doi/10.1103/PhysRevLett.77.3865

[44] I. Petousis, W. Chen, G. Hautier, T. Graf, T. D. Schladt, K. A. Persson, and F. B. Prinz, "Benchmarking density functional perturbation theory to enable high-throughput screening of materials for dielectric constant and refractive index," Phys. Rev. B, vol. 93, no. 11, p. 115151, mar 2016. [Online]. Available: https://link.aps.org/doi/10.1103/PhysRevB.93.115151

[45] H. J. Butler, L. Ashton, B. Bird, G. Cinque, K. Curtis, J. Dorney, K. Esmonde-White, N. J. Fullwood, B. Gardner, P. L. Martin-Hirsch, M. J. Walsh, M. R. McAinsh, N. Stone, and F. L. Martin, "Using Raman spectroscopy to characterize biological materials," Nature Protocols, vol. 11, no. 4, pp. 664-687, 2016.

[46] M. Wang, R. Cao, L. Zhang, X. Yang, J. Liu, M. Xu, Z. Shi, Z. Hu, W. Zhong, and G. Xiao, "Remdesivir and chloroquine effectively inhibit the recently emerged novel coronavirus (2019-nCoV) in vitro," Cell Research, no. January, pp. 2019-2021, 2020.

[47] J. Huang, J. Wen, M. Zhou, S. Ni, W. Le, G. Chen, L. Wei, Y. Zeng, D. Oi, M. Pan, J. Xu, Y. Wu, Z. Li, Y. Feng, Z. Zhao, Z. He, B. Li, S. Zhao, B. Zhang, P. Xue, S. He, K. Fang, Y. Zhao, and K. Du, "On-site detection of sars-cov-2 antigen by deep learning-based surface-enhanced raman spectroscopy and its biochemical foundations," Analytical Chemistry, vol. 93, no. 26, pp. 9174-9182, 2021, pMID: 34155883. [Online]. Available: https://doi.org/10.1021/acs.analchem.1c01061 